\title{
Ecological status of areas awarded to tribals under Forest Rights Act 2006 - a geospatial study of Adilabad Forest Division, Telangana, India
}

\author{
Deepthi Guntuka* and Sidhanand Kukrety \\ Aranya Bhavan, Saifabad, Hyderabad 500 004, India
}

Forest fragmentation and deforestation are major causes of biodiversity loss around the globe. Owing to high human and cattle population in India, the immense pressure on natural resources has led to greater degradation of natural ecosystems. The landscape-level changes have in turn resulted in changes in species diversity and composition. Adilabad district has the largest proportion of forest area in Telangana, India. We studied the impact of land right awards granted under the Recognition of Forest Rights Act (RoFR), 2006 in the Adilabad Division. We hypothesize that the forest area awarded to the tribal under RoFR and illegal encroachment in forests have adversely impacted the forest biodiversity. To verify our hypothesis, we calculated edge density (ED), patch density (PD) and core area index (CAI) of all the Reserve Forest areas using FRAGSTATS and compared the results with the pre-RoFR situation. Our results indicate that ED and PD increased predominantly across the Reserve Forest blocks where a large number of rights was granted under RoFR, whereas CAI decreased. Statistically for all the three parameters, pre- and post-RoFR values were found to be significantly different indicating that the changes due to implementation of RoFR have a significant adverse impact on the forests. Our findings are consistent with our hypothesis, and indicate that the continued use of forest areas for agriculture in RoFR and encroachment areas may further adversely impact the ecosystem. There is an urgent need to identify and implement conservation actions that would allow restoration of biodiversity of the Reserve Forest areas adversely affected by land rights awarded under RoFR. Measures such as aggregation of the scattered RoFR areas towards the Reserve Forest boundary and improved agricultural practices such as agroforestry may alleviate such adverse impacts.

Keywords: Biodiversity, core area index, edge density, Forest Rights Act, patch density.

SPREAD over $21.34 \%$ of the total geographical area, forests ${ }^{1}$ in India are home to four major biodiversity hot-

*For correspondence. (e-mail: deepthiguntuka@gmail.com) spots $^{2}$. They also support the world's second largest human population accounting for $17 \%$ and the largest cattle population in the world ${ }^{3}$. The large human and cattle populations pose immense pressure and threat to natural resources of the country mainly due to overexploitation. The anthropogenic threats are further aggravated by the poverty that exists across all the States of India. Additionally, other than anthropogenic pressure, many a times certain Federal and State policies also have an impact on the natural ecosystems. As a result of the immense biotic and abiotic pressure, most forestlands are in different stages of degradation such that only $8 \%$ of the total forests have a canopy density above $70 \%$ (ref. 1). The newly formed Telangana State (TS) has $24 \%$ of forest cover. The existing biotic and abiotic threats have further increased due to improved access of the local tribal communities by policy interventions like the Scheduled Tribes and Other Traditional Forest Dwellers (Recognition of Forest Rights) Act, 2006 (henceforth RoFR) promulgated by the Government of India in 2006. The statistics indicate predominantly high tribal population in Adilabad and Khammam districts of TS. According to the national census of 2011, Adilabad district constitutes $12.5 \%$ of the total tribal population, whereas Khammam constitutes $27.40 \%$. As such, these two districts also have the maximum number of cases awarded under RoFR.

Research indicates that degradation, deforestation and forest fragmentation, other than jeopardizing the biodiversity $^{4}$, also cause population shift, collapse of community assembly and loss of ecosystem integrity ${ }^{5}$. Forest fragmentation impacts on the species-rich terrestrial ecosystems like the forests are the greatest ${ }^{5}$. Pao and Upadhaya $^{6}$ found that the consequences of fragmentation on species diversity may cause cascading effects on the ecosystem. There is an urgent need for rational management of the remaining forests, if they are going to survive beyond the next few decades. There is a need to redefine the conservation actions that minimize the adverse impacts of anthropogenic activities on the remaining native forests. The study of these impacts and spatial patterns that exist at the landscape level provides a basis for future research on such impacts ${ }^{7}$. 


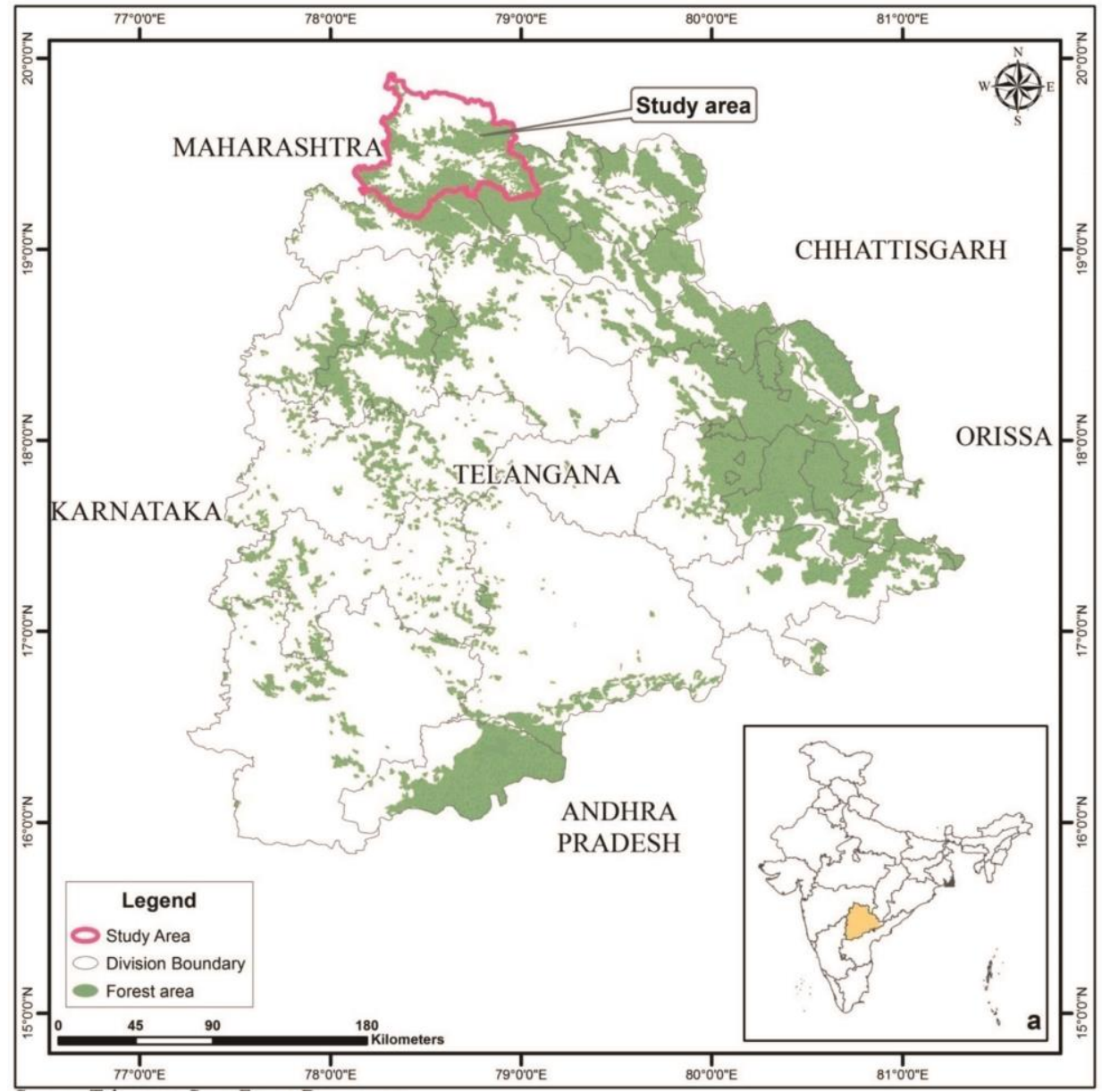

Figure 1. Map of Adilabad Division, Telangana, India, showing the study area. Source: Telangana State Forest Department.

Patch density (PD) refers to the number of patches per unit area available in a landscape. The greater the number of patches, the more fragmented the landscape; therefore, an increase in patch density may call for consideration of whether the forest has been fragmented beyond acceptable levels ${ }^{8}$. An edge effect is the effect of the juxtaposition of contrasting environments on an ecosystem. This term is commonly used in conjunction with the boundary between natural habitats, especially forests, and disturbed or developed land. Edge effects are more pronounced in small habitat fragments where they may extend throughout the patch. When an edge is created in any natural ecosystem, and the area outside the boundary is a disturbed or unnatural system, the natural ecosystem is seriously affected for some distance from the edge. Edge density (ED) refers to edge length relative to the per unit area. Edge is an important ecological concept that helps managers understand how forest harvest may affect the ecological relationship among species living in the managed areas $^{8}$.

Forest fragmentation involves the creation of 'habitat edges' and consequently the so called 'edge effects' that generally have a negative impact on the biotic and physi- cal environment. The edge effect could be defined as the interaction of two adjacent ecosystems separated through an abrupt transition. Forest fragmentation increases the amount of edges in the landscape producing important physical (e.g. radiation, moisture, temperature, wind speed and soil nutrients) and biological (e.g. species composition, competition, predation, etc.) changes along and close to the edge. The proportion of forest fragment affected by edge effects depends on the relation between fragment size and shape; the smallest and most irregularly shaped fragments are those with a greater area affected by edge effects ${ }^{4}$.

The core area is the area within a patch beyond a measured distance from the edge ${ }^{8}$. Core area index (CAI) refers to percentage of the patch that is comprised of core area. In this study, we define these areas as situated $200 \mathrm{~m}$ from an edge. The PD, ED and CAI are correlated with each other. With an increase in PD, ED also increases whereas CAI decreases.

To the best of our knowledge, consequent to the implementation of RoFR in Adilabad and Khammam districts, its impacts on the forests and biodiversity have not been studied earlier. For the purpose of this 


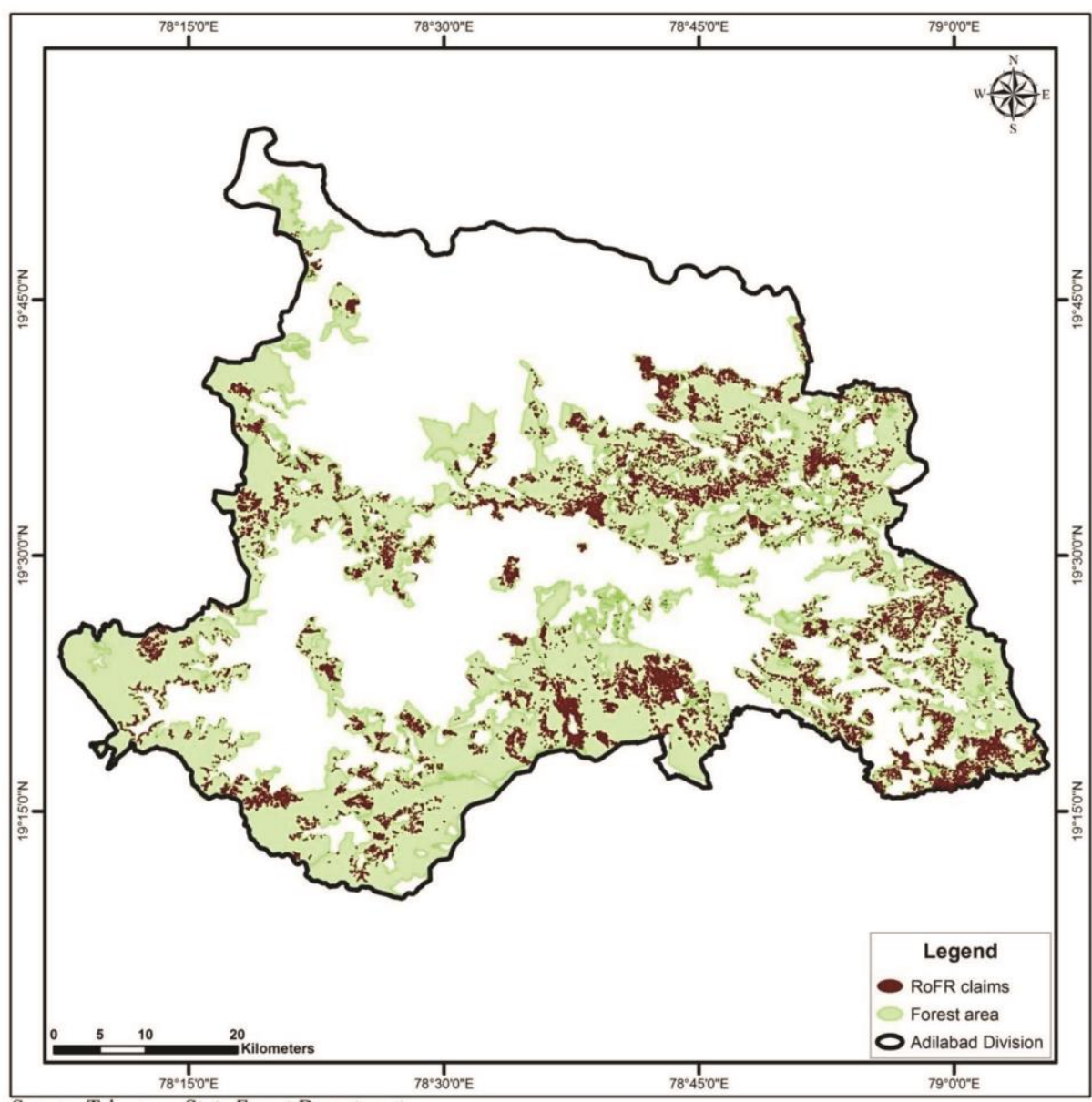

Figure 2. Map showing Recognition of Forest Rights Act area in Adilabad Division. Source: Telangana State Forest Department.

study, the pre-reorganisation boundary of Adilabad Division was taken into account (Figure 1). There are 50 forest blocks in Adilabad division, of which RoFR claims were allowed in 42 blocks (Figure 2). Keeping this in view, we took only these 42 blocks for analysis. In these 42 blocks, the proportion of RoFR areas range from a minimum of $1.29 \%$ to a maximum of $43.61 \%$, the mean being $12.09 \%$. It was observed that almost $10 \%$ of forest area was covered in RoFR. Details of RoFR lands in various Reserve Forests are provided in Supplementary Table 1.

This study aims to study and evaluate the impacts of implementation of the RoFR on Forests in Adilabad division of TS. We aim to elucidate the ecological impacts of land-use changes caused by award of forest areas to tribal communities by implementation of Recognition of Forest Rights Act (RoFR) 2006 in Adilabad District. We hypothesize that forest biodiversity in the areas awarded to the Tribal under the RoFR, has reduced when compared with the un-awarded area. We studied Patch Density (PD), Edge Density (ED), and Core Area Index (CAI)of the study area to bring out the changes in the landscape by analyzing the changes over the period of time; from 2006 to 2017. For analyses, a forest block was taken as the unit. In the context of this study a forest block is a stretch of forest land; ranging from a few hectares to large tracks that may and may not be contiguous to other forest blocks.

\section{Materials and method}

\section{Methodology}

We collected data pertaining to RoFR approved claims and forest boundaries from the Telangana State Forest Department. The RoFR data were converted into spatial format using MS Excel (Microsoft Corporation ${ }^{\odot}$ 2007) and ArcGIS $\left(\text { ESRI }^{\odot} 2016\right)^{9}$. Thereafter, using geospatial tools we studied the changes in forest and recorded the net impact of implementation of RoFR. For this, two separate output files, viz. pre-RoFR and post-RoFR files were created. FRAGSTATS ${ }^{10}$ was used to estimate the changes in ED, PD and CAI. The CAI results were divided into three categories: $0 \%-15 \%$ - high, 15\%$30 \%$ - medium and $>30 \%-$ low. ED was also divided into three categories: $0 \%-15 \%-$ low, 15\%-30\%-medium and $>30 \%$-high. Thereafter, two-paired Z-test was 


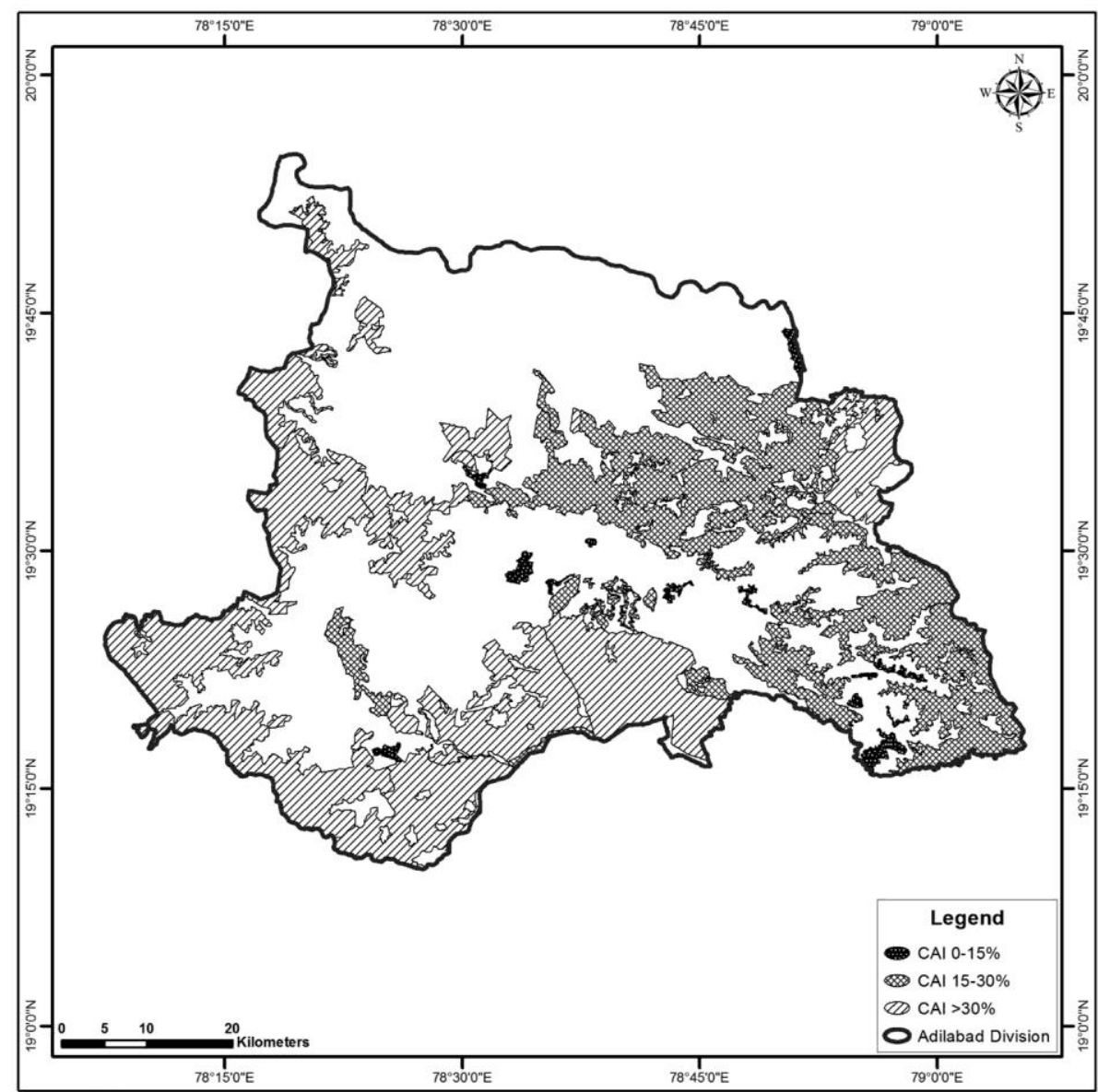

Figure 3. Map showing post-RoFR core area index category in Adilabad Division. Source: Telangana State Forest Department.

Table 1. Pre- and post-Recognition of Forest Rights Act (RoFR) patch density (PD) of Adilabad Division, Telagana, India

\begin{tabular}{lcc}
\hline PD & Pre-RoFR & Post-RoFR \\
\hline$N$ & 42 & 42 \\
Minimum & 0.003 & 0.158 \\
Maximum & 0.910 & 32.046 \\
Mean & 0.218 & 3.236 \\
Median & 0.131 & 1.658 \\
SD & 0.240 & 5.448 \\
$P$ & \multicolumn{2}{c}{0.0007} \\
\hline
\end{tabular}

Source: Supplementary Table 2.

Table 2. Pre- and post-RoFR edge density (ED) of Adilabad Division

\begin{tabular}{lcc}
\hline ED & Pre-RoFR & Post-RoFR \\
\hline$N$ & 42 & 42 \\
Minimum & 0.000 & 3.025 \\
Maximum & 19.726 & 76.214 \\
Mean & 2.229 & 22.871 \\
Median & 0.772 & 19.365 \\
SD & 3.914 & 16.389 \\
$P$ & \multicolumn{2}{c}{0.000} \\
\hline
\end{tabular}

Source: Supplementary Table 3.
Table 3. Pre- and post-RoFR core area index (CAI) of Adilabad Division

\begin{tabular}{lcc}
\hline CAI & Pre-RoFR & Post-RoFR \\
\hline$N$ & 42 & 42 \\
Minimum & 0.402 & 0.000 \\
Maximum & 90.224 & 64.102 \\
Mean & 50.688 & 27.229 \\
Median & 52.015 & 27.247 \\
SD & 23.635 & 19.138 \\
$P$ & & 0.000 \\
\hline
\end{tabular}

Source: Supplementary Table 4.

performed to ascertain whether the post-RoFR mean values of indicators were significantly different from the pre-RoFR values or not.

\section{Results and discussion}

This study indicates loss of biodiversity in the forest areas diverted for agriculture by the tribal communities under the RoFR Act, 2006. 


\section{Patch density}

FRAGSTATS quantifies the spatial distribution of patches on a block in a landscape. Results indicate that PD increased in all the 42 blocks, while it was above the mean value in 25 forest blocks. Minimum PD was 0.003-0.158 and maximum was 0.910-32.046. There was a direct correlation between PD and degree of disturbance. Higher the value of PD, higher was the disturbance magnitude and vice versa ${ }^{6}$. After calculating the two-paired $Z$-test, $Z$ value (3.129) for this standard normal curve area is 0.9993. Paired $Z$-test indicated that the post-RoFR mean value of PD was significantly different from the pre-RoFR value $(P=0.0007)$.

\section{Edge density}

FRAGSTATS results showed minimum ED (0.00) in 14 out of 42 blocks during the pre-RoFR period, whereas maximum ED recorded was 19.73. Post-RoFR FRAGSTATS recorded minimum ED of 3.02 and maximum of 76.21. This indicates drastic increase in ED in all the 42 blocks, highlighting severe fragmentation of the area.

Edge effect plays a key role in fragment dynamics and the matrix has a major influence on fragment connectivity and functioning ${ }^{11}$. The increased ED suggests that postRoFR, fragmentation of ecological landscape has increased manifold due to the presence of agriculture matrix resulting in poor connectivity between the patches leading to loss of ecosystem function and biodiversity. After calculating the two-paired $Z$-test, $Z$-value (4.90) for this standard normal curve area is 0.99999971. Paired Ztest showed that the post-RoFR mean value of ED was noticeably different from the pre-RoFR value $(P=0.000)$.

Core area index: In this study CAI was calculated by taking the depth as $200 \mathrm{~m}$. The output indicated decrease in core area based on Table 3, minimum CAI $0.402 \%$ $0.000 \%$. For the Jainoor and Mahagoan forest blocks, CAI was zero. FRAGSTATS indicated $26 \%$ reduction in pre-and post-RoFR CAI, i.e. from 90.224 to 64.102, whereas the mean reduction was $46 \%$, i.e. from 50.688 to 27.229.

Reduced core area indicates more edges, highlighting severe fragmentation of the forest landscape. Lower CAI scores are useful indicators of forest patches at risk, and therefore the reduction in CAI indicates loss of habitable area leading to biodiversity loss. After calculating the two-paired $Z$-test, $Z$-value (5.33) for this standard normal curve area is 0.00000029 . Paired $Z$-test showed that the post-RoFR mean value of CAI was considerably different from the pre-RoFR value $(P=0.000)$.

From the results it is evident that the extent of fragmentation has increased in the forest landscape on account of RoFR in Adilabad Division. It is also reflected in the increased number of patches in the Division. Edge is likely to continue to increase in this landscape in future due to extensive agriculture and other land-use practices. As seen in Figure 3, the eastern part of Adilabad Division is likely to become highly fragmented leading to reduction in the core area. Our results indicate that 13 of the 42 blocks would become highly prone to reduction in the core area $(\mathrm{CAI}=0 \%-15 \%), 11$ blocks will be medium prone $(\mathrm{CAI}=15 \%-30 \%)$, and 18 blocks will be less prone to reduction in the core area (CAI >30\%) (Figure 3 ). This indicates that more than $50 \%$ of the blocks are more likely to get fragmented in due course. This has huge implications on the overall productivity, biodiversity and community structure of the forests in the area.

If further diversion of forest area continues in some of these forest blocks, the entire core area is likely to vanish. Reducing further diversions of the forest areas while simultaneously improving the conservation holds key for improving biodiversity of these forest blocks. Julieta and Víctor $^{4}$ note that in an event of modification of the forest edge beyond the range of natural intrinsic variation, the portion of the fragment under the influence of edge effects will be unsuitable for the original ecosystem. Consequently, from the conservation point of view these fragmented areas are permanently lost. Therefore, in the areas awarded under the RoFR Act, 2006, the changes in the physical environment caused by edges may directly affect forest structure. The creation of an edge increases the incident light which, in turn, promotes plant growth. Thus, even several decades after creation of the edge, forest structure near it remains changed.

\section{Conclusion}

We studied the impact of implementation of RoFR Act on forest areas in Adilabad Forest Division, TS. This was accomplished by evaluating the pre- and post-RoFR ED, $\mathrm{PD}$ and CAI. These indices were calculated for all the 42 forest blocks using FRAGSTATS, where the RoFR areas were awarded to the local tribals. Our results indicate overall degradation of all the three ecological indices in the RoFR areas, showing changes in biotic and abiotic conditions and loss of biodiversity. Temporal estimates of the intensity and impact of RoFR are urgently required to evaluate the adverse impacts of this Act in other districts. Owing to extensive agriculture and other non-forestry land-use-practices, edge will continue to increase in future landscapes. There is a critical requirement of personalized conservation approach on part of the Forest Department. For instance, all the RoFR areas scattered over a Reserve Forest block could be segregated towards the boundary, so that honeycombing of the Reserve Forest could be avoided. This in turn will reduce the impact of RoFR diversion of these areas. Consequently, the 
Reserve Forest block may retain its biodiversity for a longer time. Secondly, participatory management of these areas like joint forest management might help in providing livelihood to the tribal populations, while also protecting the forests.

1. http://fsi.nic.in (accessed on 17 May 2017)

2. http://bsienvis.nic.in/files/Biodiversity\%20Hotspots\%20in\%20India. pdf (accessed on 18 May 2017).

3. http://dahd.nic.in/sites/default/files/BAH_\%26_FS_Book.pdf (accessed on 18 May 2017)

4. Julieta, B. M. and Víctor, A. R., Habitat fragmentation, edge effects and biological corridors in tropical ecosystems, 2008.

5. Mikkelson, G. M., How do food webs fall apart? A study of changes in trophic structure during relaxation on habitat fragments. Oikos, 1993, 67, 539-547.

6. Pao, T. and Upadhaya, K., Effect of fragmentation and anthropogenic disturbances on floristic composition and structure of subtropical broad leaved humid forest in Meghalaya, Northeast India. Appl. Ecol. Environ. Res., 2017, 15, 385-407; doi:10.15666/aeer/ 1504_385407.

7. Singh, K. R., Mapping and quantitative assessment of vegetation of Jiribam Sub-Division, Imphal East District, Manipur, India using remote sensing and GIS. Ph D thesis, Department of Ecology and Environmental Science, Assam University, Silchar, 2015; http://shodhganga.inflibnet.ac.in/handle/10603/54555 (accessed on 23 August 2017).

8. Perera, A. H., Euler, D. L. and Thompson, I. D., Ecology of a Managed Terrestrial Landscape: Patterns and Processes of Forest Landscapes in Ontario, UBC Press, University of British Columbia, Canada, 2001.

9. ESRI 2016. ArcGIS Desktop: Release 10.5 Redlands, CA: Environmental Systems Research Institute; https://www.esri.com/ en-us/home (accessed on 19 May 2017)

10. McGarigal, K., Cushman, S. A. and Ene, E., FRAGSTATS v4: Spatial Pattern Analysis Program for Categorical and Continuous Maps. Computer software program produced at the University of Massachusetts, Amherst, 2012; http://www.umass.edu/landeco/ research/fragstats/fragstats.html (accessed on 19 May 2017).

11. Laurance, W. F. et al., Ecosystem decay of Amazonian forest fragments: a 22-year investigation. Conserv. Biol., 2002, 16, 605618; doi:10.1046/j.1523-1739.2002.01025.x (accessed on 16 August 2017)

ACKNOWLEDGEMENT. We thank the Telangana State Forest Department for providing forest block information and details regarding RoFR claims for the study.

Received 6 March 2018; revised accepted 16 May 2019

doi: $10.18520 / \mathrm{cs} / \mathrm{v} 117 / \mathrm{i} 3 / 434-439$ 